\title{
Vibrational properties of single-wall nanotubes and monolayers of hexagonal BN
}

\author{
D. Sánchez-Portal* \\ Centro Mixto CSIC-UPV/EHU and Donostia International Physics Center (DIPC), Paseo Manuel de Lardizabal 4, \\ 20018 Donostia-San Sebastian, Spain \\ E. Hernández ${ }^{\dagger}$ \\ Institut de Ciència de Materials de Barcelona, ICMAB-CSIC, Campus de Bellaterra, 08193 Bellaterra, Barcelona, Spain
}

(Received 18 September 2002; published 20 December 2002)

\begin{abstract}
We report a detailed study of the vibrational properties of $\mathrm{BN}$ single-walled nanotubes and of the $\mathrm{BN}$ monolayer. Our results have been obtained from a well-established tight-binding model complemented with an electrostatic model to account for the long-range interactions arising from the polar nature of the material and which are not included in the tight-binding model. Our study provides a wealth of data for the BN monolayer and nanotubes, such as phonon band structure, vibrational density of states, elastic constants, etc. For the nanotubes we obtain the behavior of the optically active modes as a function of the structural parameters, and we compare their frequencies with those derived from a zone-folding treatment applied to the phonon frequencies of the BN monolayer, finding general good agreement between the two.
\end{abstract}

DOI: 10.1103/PhysRevB.66.235415 PACS number(s): 63.22. +m, 62.25.+g, 78.30.Na, 78.20.Bh

\section{INTRODUCTION}

The discovery of the fullerenes ${ }^{1}$ in the mid-1980s and, especially, that of carbon nanotubes ${ }^{2}$ in the early 1990 s has fueled a frenzy of research activity in the field of carbon nanostructures, doubly motivated by their large potential for practical applications, on the one hand, and because these systems constitute a new open field for basic research at the nanoscale on the other. Nanotubes can be single walled or multiwalled, and are quasi-one-dimensional (quasi-1D) structures having large aspect ratios (the quotient of length over diameter). Single-wall carbon nanotubes (SWNT's) can be either semiconducting or metallic, depending on their structural parameters, and can be chiral or achiral. They have large thermal conductivity and have the highest Young's modulus ever measured. ${ }^{3-6}$ Nanotubes can be filled with other elements, compounds or even fullerenes in their internal channel, forming structures similar to nanoscale coaxial cables. All these properties confer on nanotubes a large potential for technological applications, some of which have already been demonstrated. Nanotubes have been used to fabricate field emission devices, ${ }^{7}$ tips for scanning probe microscopy instruments, ${ }^{8}$ constituents of nanoelectronic devices, ${ }^{9}$ gas sensors, ${ }^{10}$ composite reinforcement, ${ }^{11}$ lubrication, etc. It is therefore not surprising that these systems have been and continue to be the focus of considerable interest; the large number of review articles ${ }^{12}$ and monographs ${ }^{13}$ devoted to the different aspects of fullerenes and nanotubes in recent years provides further proof of this interest.

One of the probes most frequently used to characterize carbon nanotube samples is vibrational spectroscopy and, specifically, Raman spectroscopy. ${ }^{14}$ Raman spectroscopy offers experimentalists a rapid way of estimating the diameter distribution of tubes present in a sample, because the lowfrequency radial breathing mode (RBM) of SWNT's has a frequency which is inversely proportional to the square of the nanotube diameter, independently of the nanotube structure, determined by the nanotube indices $(n, m)$. Also, the high-frequency $G$-band modes resulting from atomic vibrations in the nanotube shell can help to distinguish between metallic and semiconducting nanotubes in the sample. ${ }^{15}$ Jorio and co-workers ${ }^{16}$ have recently demonstrated the ability of Raman spectroscopy to provide full structural determinations of isolated carbon SWNT's. The diverse aspects of phonons in carbon SWNT's and the use of Raman spectroscopy as a tool for their characterization have been recently reviewed by Dresselhaus and Eklund. ${ }^{14}$

Soon after the discovery of carbon nanotubes it was speculated that other materials could possibly form similar nanostructures, since there are several elements and many compounds which form layered structures bearing some resemblance to graphite. The most obvious candidate was hexagonal $\mathrm{BN}(h-\mathrm{BN})$, which was predicted on the basis of theoretical calculations ${ }^{17,18}$ to be capable of forming nanotubes, a prediction which was later corroborated experimentally by the synthesis of such nanotubes. ${ }^{19}$ Today we know that many other structures can form nanotubes $\left(\mathrm{MoS}_{2}\right.$, $\left.\mathrm{WS}_{2},{ }^{20} \mathrm{Bi}^{21} \ldots\right)$; nevertheless, it is still the case that carbon nanotubes continue to attract a larger interest, but certainly these other structures are interesting in their own right and may be able to offer different possibilities for technological applications that carbon nanotubes cannot provide. Both multiwalled and single-walled ${ }^{22} \mathrm{BN}$ nanotubes can now be readily synthesized, and these tubes are uniformly insulating, tending to have a zigzag structure.

As other types of nanotubes become more common, many of the characterization tools and techniques extensively used in the case of carbon nanotubes will undoubtedly also find application in the study of these other structures. In particular, vibrational spectroscopy, which has proved to be such a useful tool in the case of carbon nanotubes, is likely to prove useful also in these cases. In this context, it is interesting to consider the phonon properties of $\mathrm{BN}$ nanotubes from a theoretical point of view; their study will help to develop the characterization potential of spectroscopic techniques when they are applied to $\mathrm{BN}$ nanotubes. The aim of this paper is to 
provide such a theoretical study. We report extensive theoretical calculations of the vibrational properties of a $h$-BN layer and of a number of $\mathrm{BN}$ nanotubes having diameters in the range of $0.4-2 \mathrm{~nm}$, including zigzag, armchair, and several chiral nanotubes. From our results for the flat sheet we have performed a zone-folding analysis in order to predict the vibrational properties of the tubular structures from those of the flat layer, a technique which has been frequently used in the case of carbon nanotubes. The direct calculation of the vibrational properties of tubular structures allows us to compare the predictions of zone folding with the actual results and thus gauge the applicability of the zone-folding approach for BN nanotubes. Other authors have previously considered the vibrational ${ }^{23}$ and elastic ${ }^{24}$ properties of isolated $h$-BN monolayers and of bulk $h$-BN. ${ }^{25,26}$ Some aspects of the elasticity of BN nanotubes have also been studied in Refs. 27 and 24. However, the study of the vibrational properties in the case of BN nanotubes has not, to our knowledge, been addressed to date.

The structure of the paper is as follows. In Sec. II we describe the model and calculation procedure used in our study; later in Sec. III we discuss our findings, starting with a thorough description of the vibrational properties of an isolated $h$-BN monolayer, followed by the results obtained for the nanotubes, establishing a comparison between the results obtained from the zone-folding analysis and those obtained from direct calculation. Our summary and conclusions are discussed in Sec. IV.

\section{MODEL AND COMPUTATIONAL PROCEDURE}

All calculations have been performed using the nonorthogonal tight-binding ${ }^{28}$ (TB) parametrization of Widany et al. ${ }^{29}$ This is a parametrization following the scheme proposed by Porezag and co-workers. ${ }^{30}$ This model employs a basis set consisting of one $S$ and three $P$ functions per atom, to represent valence states, and the resulting Hamiltonian and overlap matrix elements extend up to a range of approximately $5 \AA$. The core-core repulsion is modeled by means of a simple pair-repulsive potential. The original work of Porezag et $a l .{ }^{30}$ described a non-self-consistent TB model; although an extension of the methodology has been proposed which incorporates some degree of self-consistency, ${ }^{31}$ here we only have used the model of Widany et al. ${ }^{29}$ in its nonself-consistent form. It is worth commenting that in spite of its simplicity this model has proved to be quite successful. ${ }^{29,27}$

The equilibrium structures of the tubes were obtained by careful minimization of the total energy with respect to both the atomic coordinates and the lattice constant along the tube axis. For the phonon calculations, we then computed the force-constant matrix in real space using a finite-difference approach. ${ }^{32}$ We used atomic displacements of $0.02 \AA$, and the force constants were taken as the average of the results obtained with positive and negative displacements, in order to eliminate anharmonic effects. Since all nitrogen and boron atoms are equivalent by symmetry in the tubes, we only calculated the force constants for one nitrogen and one boron atom in the supercell and generated the rest of the matrix using the symmetry operations.

The force constants have to be calculated between a given atom and all the other atoms in the system. However, since the interactions in our TB model decrease rapidly with distance, only the elements with atoms sufficiently close have to be computed. To do so, we set up a supercell large enough that a sufficient number of neighbors is included. It must be kept in mind that, in the supercell geometry, a given atomic displacement in the central cell is always accompanied by the same displacement of all image atoms. We need, therefore, to use a supercell such that the effect of the image displacements is negligible. For this purpose we have used supercells containing six unit cells in the case of $(n, n)$ and $(n, 0)$ tubes and two and one unitcells, respectively, in the case of the $(10,5)$ and $(10,7)$ tubes. The supercells contain hence a minimum of 144 atoms for the $(6,0)$ and $(6,6)$ tubes and a maximum of 384 atoms in the case of the $(16,0)$ tube.

The above-described TB model does not by itself incorporate any long-range electrostatic interactions. Such interactions are important when considering the vibrational properties of polar materials, because they will make a long-range contribution to the force constants. Therefore we have opted for correcting this shortcoming in the TB model in a physically sound but otherwise empirical way. The key observation in order to introduce the effects of the electrostatic interactions is that, when an atom $i$ suffers a displacement $\mathbf{u}(i)$ from its equilibrium position, a net electric dipole of magnitude $\mathbf{p}^{i}=\Sigma_{\mu} Z_{\nu \mu}^{i} u_{\mu}^{i}$ appears associated with this atomic movement. ${ }^{33}$ Here $Z_{\nu \mu}^{i}$ is the Born effective charge tensor ${ }^{33}$ of the atom $i$. As a consequence, if two atoms $i$ and $j$ are simultaneously displaced from their positions, besides the interaction energy given by our TB model, it is necessary to include the long-range interaction between the two electric dipoles generated. This gives rise to a new term in the forceconstant matrix of the form ${ }^{33}$

$$
C_{\nu \mu}^{L R}\left(\mathbf{r}_{i j}\right)=f\left(r_{i j}\right) \sum_{\nu^{\prime} \mu^{\prime}} \frac{Z_{\nu \nu^{\prime}}^{i} Z_{\mu \mu^{\prime}}^{j}}{\epsilon}\left(\frac{\delta_{\nu^{\prime} \mu^{\prime}}}{r_{i j}^{3}}-3 \frac{r_{i j \nu^{\prime}} r_{i j \mu^{\prime}}}{r_{i j}^{5}}\right),
$$

where the superindex $L R$ in $C_{\nu \mu}^{L R}\left(\mathbf{r}_{i j}\right)$ indicates that this is the long-range contribution to the force constants; $f\left(r_{i j}\right)$ is a switching function (see below), $\epsilon$ is the dielectric constant, $\mathbf{r}_{i j}$ is the vector going from atom $i$ to atom $j$, and $r_{i j}$ is the distance between these two atoms. The switching function is designed to come into play at distances sufficiently large so as to not affect the TB model; it takes the form

$$
f(r)=1-e^{-\left(r / r_{c}\right)^{3}} .
$$

Following the usual approach, ${ }^{33}$ it is also necessary to modify the on-site elements of the force matrix in order to satisfy the acoustic sum rule. Therefore, we take

$$
C_{\nu \mu}^{L R}\left(\mathbf{0}_{i i}\right)=-\sum_{j \neq i} C_{\nu \mu}^{L R}\left(\mathbf{r}_{i j}\right) .
$$

The Born effective charge tensor has been taken from recent $a b$ initio density functional calculations: ${ }^{26} Z_{\perp}^{B}=-Z_{\perp}^{N}=0.82$ 


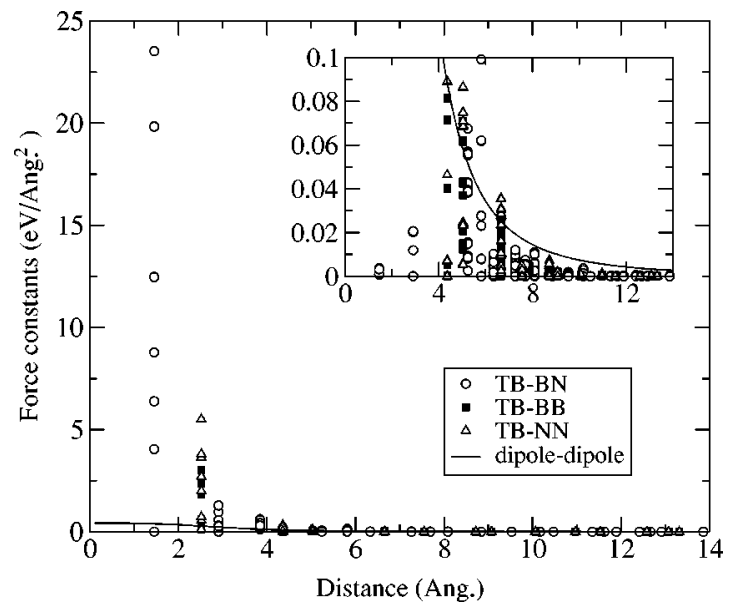

FIG. 1. Force constants derived from the TB model (symbols) and the typical behavior of the electrostatic interactions [approximated in the plot by $\left.f(r) Z_{\|}^{2} /\left(\epsilon r^{3}\right)\right]$ (solid line), as a function of distance. The inset shows more clearly the behavior for large distances.

for out-of-plane and $Z_{\|}^{B}=-Z_{\|}^{N}=2.71$ for in-plane displacements. ${ }^{34}$ We have taken $\epsilon=4$ and $r_{c}=2.6 \AA$, the distance of separation between second nearest neighbors in the $h$-BN layer. We have not attempted to fit these values in order to improve the agreement of our results with experimental measurements. Rather, our approach has been to take reasonable values for them in order to estimate the effect of the polar interactions on the vibrational properties of the BN monolayer and in the nanotubes. Our chosen value for $\epsilon$ is close to the experimental one for $h$-BN (4-5 depending on the direction $\left.{ }^{35,26}\right)$, and the value of $r_{c}$ used in Eq. (2) is motivated by the fact that force constants arising from the TB model alone decay very rapidly with distance, and they are already very small at this value of $r_{c}$, as can be seen in Fig. 1. By choosing $r_{c}$ equal to the second nearest-neighbor distance we ensure a smooth switching between the shortrange TB model and the long-range electrostatic one, with minimum interference between them.

Once we have the force-constant matrix in real space, we can calculate the dynamical matrix in reciprocal space and diagonalize it to obtain the phonon modes and frequencies. In the case of bulk polar systems the computation of the dynamical matrix in reciprocal space has to be made with some care. The reason is that, due to the long-range nature of the dipole-dipole interaction in Eq. (1), a different limit for the dynamical matrix is obtained, as the phonon wave vector $\mathbf{k}$ approaches $\boldsymbol{\Gamma}$, in the cases of longitudinal and transversal vibrations. In our case however, due to the reduced dimensionality of the systems considered (a monolayer and singlewalled tubes), the Fourier transform of the dynamical matrix can be performed without further complications by simply adding up the elements of the real-space matrix with the appropriate phase factors. Given its simple analytical form, we can include a large number of neighbors (we typically take all neighbors within a radius of $\sim 200 \AA$ ) in the summation involving the dipole-dipole interactions and guarantee a good convergence. The same limit is found for both polarizations when $\mathbf{k} \rightarrow \boldsymbol{\Gamma}$, and there are no splittings between in-plane longitudinal and transversal optical modes at $\boldsymbol{\Gamma}$ (see the Appendix).

\section{RESULTS AND DISCUSSION}

One of the aims of this work is to judge the validity of the zone-folding approach for obtaining phonon information of tubular structures from the phonon band structure of the flat sheet, a procedure which has been frequently used in the case of carbon nanotubes. ${ }^{14}$ To this end we have performed a phonon analysis of the flat sheet and of several nanotubes (armchair, zigzag, and chiral) so as to compare the zonefolding results with those obtained directly from the tubular structures. In the following we will first present the results obtained for the flat sheet, followed by those for a selected set of nanotubes. The comparison between these latter results and the zone-folding predictions will be then presented.

\section{A. Flat sheet}

Using the TB model discussed in Sec. II, with and without the dipolar interactions, with a supercell containing 192 atoms we have obtained the phonon dispersion curves and vibrational density of states for a flat sheet of $h$-BN, plotted in Fig. 2. In order to check the convergence of the results with respect to the supercell size, we performed a test with a supercell containing 320 atoms; the results obtained with the smaller cell are indistinguishable from those of the larger cell, and hence all subsequent analysis was performed with the 192-atom cell results. We also performed a more detailed analysis of the convergence of the phonon bands with respect to the lower threshold of the elements of the real-space forceconstant matrix included in the construction of the dynamical matrix. We focus first on the convergence with respect to the TB force constants. We found that including all the couplings coming from the TB Hamiltonian with absolute value larger than $1.2 \times 10^{-2} \mathrm{eV} / \AA^{2}$ was necessary to adequately converge all bands. This threshold corresponds to a spatial cutoff of $6.7 \AA$, which includes all atoms within the tenth nearestneighbor shell. This large cutoff is required to correctly reproduce the quadratic behavior of the lowest acoustic band (labeled ZA in Fig. 2) in the limit $\mathbf{k} \rightarrow \boldsymbol{\Gamma}$, with a zero sound velocity (slope of the band) as $\mathbf{k}$ approaches $\boldsymbol{\Gamma}$. This quadratic behavior of the ZA band is a consequence of the fact that, at least to the lowest order in its amplitude, the strain energy created by this vibration is solely associated with the curvature that this out-of-plane bending mode induces in the layer. Optical bands typically converge much faster (ca. including interactions with neighbors within the third or fourth nearest-neighbor shell). It is worth mentioning in this respect that in the earlier theoretical work of Miyamoto et al. ${ }^{23}$ a supercell which only permitted the inclusion of second nearest-neighbor interactions was used due to computational limitations (Miyamoto and co-workers used first-principles methods, which are significantly more costly, to evaluate the force-constant matrix). Although inclusion of up to second nearest-neighbor interactions is sufficient to adequately converge most bands, it fails to reproduce the overbending (see 

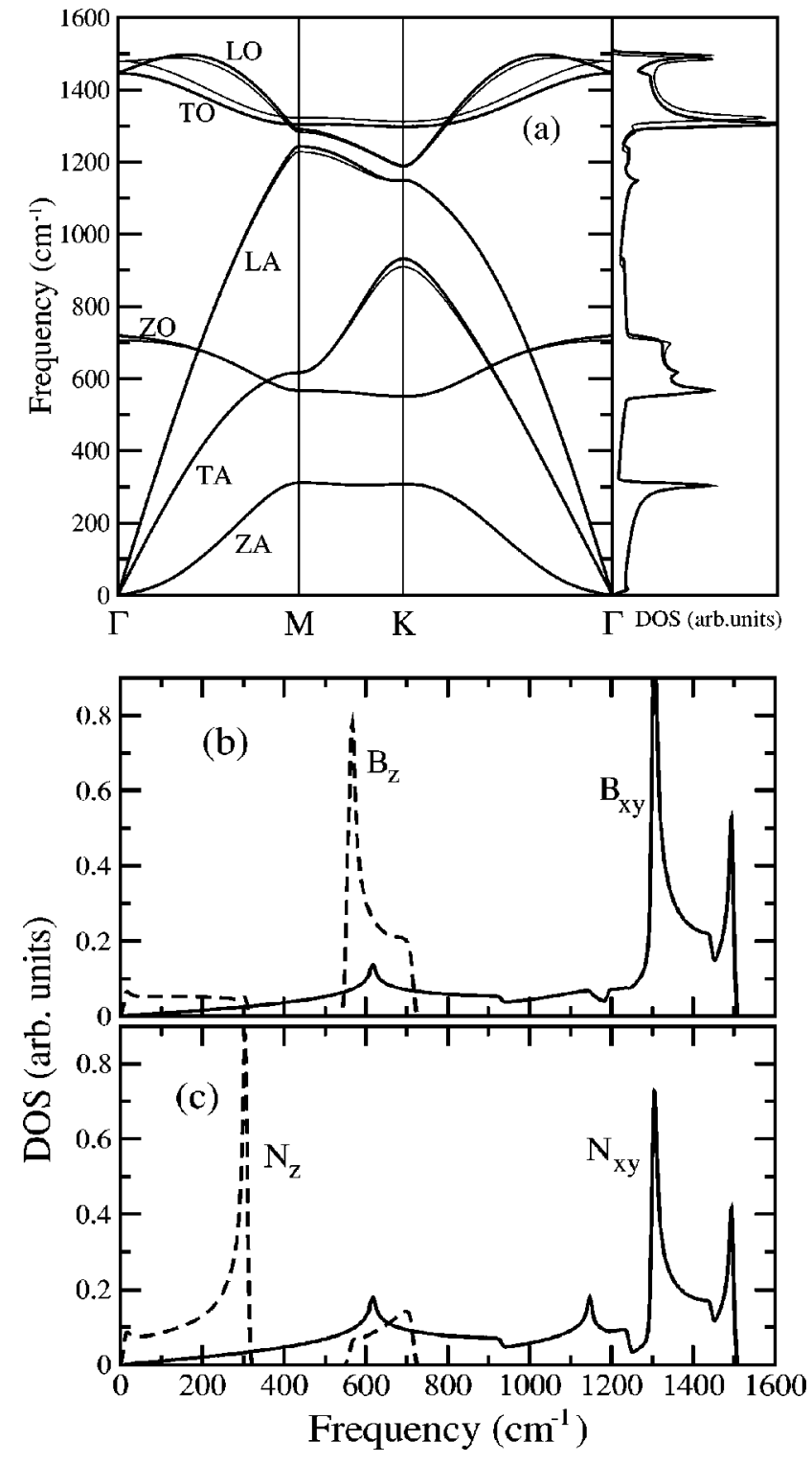

FIG. 2. (a) Phonon band structure of the BN hexagonal monolayer (right) and the corresponding density of vibrational states (left); thick lines correspond to band structure and density of states calculated with electrostatic interactions included, while thin lines give results obtained only with the TB model. (b) Projected density of states on the B atoms; the solid line represents the density of states projected onto the plane of the monolayer, while the dashed line gives the density of states projected perpendicular to the plane of the monolayer. (c) As in (b) for the $\mathrm{N}$ atoms.

below) of the longitudinal-optical (LO) band, for which the maximum occurs not at $\boldsymbol{\Gamma}$ but approximately halfway along the symmetry lines $\boldsymbol{\Gamma}-\mathbf{M}$ and $\boldsymbol{\Gamma}-\mathbf{K}$, and is insufficient to reproduce the quadratic behavior of lowest acoustic band around $\boldsymbol{\Gamma}$.

Let us now comment briefly on the convergence with respect to the long-range electrostatic interactions. As already commented in the previous section, due to its simple analytical form, we have used a large cutoff of $200 \AA$ for the dipoledipole interactions. However, we have checked that already with a cutoff of $\sim 25 \AA$ the results are well converged. Fur- ther reducing the interaction range starts to have an appreciable effect. Not only the position of the optical branches at and close to $\boldsymbol{\Gamma}$ start to shift from their converged values, but the finite slope at $\boldsymbol{\Gamma}$ that the LO mode must exhibit as a consequence of the long-range nature of the interactions also disappears (see the Appendix A).

As can be appreciated in Fig. 2, due to the twodimensional character of the system, there is a separation between in-plane and out-of-plane modes, the latter being the lower-frequency ones, with a maximum at $\boldsymbol{\Gamma}$ of $719 \mathrm{~cm}^{-1}$ with dipolar interactions included $\left(706 \mathrm{~cm}^{-1}\right.$ if these are not included). This frequency separation is to be expected, since in-plane modes excite both bond stretchings (hard) and bond bendings (soft), while out-of-plane modes result mostly in bond-bending motion and very little bond stretching. The highest-frequency bands, the LO and the transversal-optical (TO), have a frequency at $\Gamma$ of $1446 \mathrm{~cm}^{-1}$ when the dipolar interactions are included $\left(1479 \mathrm{~cm}^{-1}\right.$ without). The maximum of the LO band occurs not at $\boldsymbol{\Gamma}$, but at an intermediate point away from the Brillouin zone edges, as pointed out above. This is known as overbending, and the presence of overbending in the flat sheet phonon band structure can have interesting consequences for the phonon band structure of the nanotubes, as it may lead to the appearance of new modes at $\boldsymbol{\Gamma}$ having higher frequencies in the tubular structures than in the flat sheet. ${ }^{36}$ Note that including the dipolar interactions increases the overbending, which is much smaller when the electrostatics are not appropriately accounted for. Another noteworthy characteristic of the phonon band structure displayed in Fig. 2(a) is the splitting of approximately $250 \mathrm{~cm}^{-1}$ existing between the $\mathrm{ZO}$ and ZA bands along M-K. In a homopolar hexagonal sheet such as graphene these two bands cross at $\mathbf{K} .^{37}$

From the slopes of the transversal-acoustic (TA) and longitudinal-acoustic (LA) bands in the limit $\mathbf{k} \rightarrow \boldsymbol{\Gamma}$ we deduce a sound velocity of $13 \mathrm{~km} / \mathrm{s}$ and $21 \mathrm{~km} / \mathrm{s}$, respectively. As already pointed out above, the ZA band exhibits a $k^{2}$ behavior in this limit, as expected, and therefore the sound velocity associated with this band is zero. Fitting the frequencies below $250 \mathrm{~cm}^{-1}$ to the expression $\omega=2 \pi \nu=\delta k^{2}$ $+\gamma k^{3}$, we can estimate a value of $\delta \approx 1820 \pm 60 \mathrm{~cm}^{-1} \AA^{2}$ $=5.5 \pm 0.2 \times 10^{-7} \mathrm{~m}^{2} \mathrm{~s}^{-1}$. This parameter is interesting because it allows us to estimate the energy necessary to roll up the BN sheet in order to form the nanotubes. Simple arguments show that the strain energy per atom can be approximated by $E_{s t}=C / r^{2}$ with $C=\delta^{2}\left(m_{N}+m_{B}\right) / 4, m_{N}$ and $m_{B}$ being the masses of the nitrogen and boron atoms, respectively, and $r$ the tube radius. The value obtained for $C$ in this way is $1.91 \mathrm{eV} \AA^{2}$, which is in reasonable agreement with the magnitude obtained from total energy calculations performed using both a tight-binding Hamiltonian similar to the one utilized here ${ }^{27}$ and first-principles density functional calculations. ${ }^{18,24}$ A similar estimation in the case of graphene $^{37}\left(\delta \approx 6 \times 10^{-7} \mathrm{~m}^{2} \mathrm{~s}^{-1}\right)$ leads to a value $C$ $\approx 2.3 \mathrm{eV} \AA^{2}$. Therefore, already looking at the phonon band structures it is possible to find an indication that the strain energy is smaller in the case of BN tubes than for carbon nanotubes, a result which is confirmed by more sophisticated calculations. ${ }^{18,27,24,38}$ 
TABLE I. Elastic constants and Young modulus $Y$, in units of TPanm, and Poisson ratio $\sigma$ of the flat $\mathrm{BN}$ hexagonal sheet, as calculated directly from the force-constant matrix given by our model. The results obtained from the sound velocities of the TA and LA bands [see Eq. (5)] are shown in brackets.

\begin{tabular}{lc}
\hline \hline$C_{x x x x} \equiv C_{11}$ & $0.343(0.322)$ \\
$C_{x x y y} \equiv C_{12}$ & 0.119 \\
$C_{x y x y} \equiv C_{66}$ & $0.107(0.123)$ \\
$Y$ & 0.302 \\
$\sigma$ & 0.347 \\
\hline \hline
\end{tabular}

Using the calculated force-constants matrix we can directly obtain the elastic constants ${ }^{39}$ of the flat sheet, which are given in Table I. In bulk three-dimensional systems it is customary to quote elastic constants in units of pressure, due to the inverse equilibrium cell volume factor that appears in the definition of the elastic constants. ${ }^{39,40}$ However, in twodimensional systems the definition of the cell volume is arbitrary, and it is therefore more appropriate to use cell area in the definition of the elastic constants; ${ }^{27}$ hence, we provide our results in units of pressure times length. Comparing with previous calculations in the literature, our result for $C_{x x x x}$ is in reasonable agreement with the value of $0.271 \mathrm{TPa} \mathrm{nm} \mathrm{ob-}$ tained for the in-plane stiffness ${ }^{41}$ by Kudin and co-workers using density-functional theory and Gaussian-type orbitals. ${ }^{24}$ Our result is also in quite good agreement with the value of $0.309 \mathrm{TPa} \mathrm{nm}$ that can be deduced from the plane-wave density-functional calculation of the elastic constants of bulk $h$-BN reported by Ohba et al. in Ref. 26 (we use here the calculated interlayer distance of $3.25 \AA$ to translate the data from the bulk to the monolayer geometry). However, the comparison is poorer for the $C_{x y x y}$ elastic constant, for which the data of Ohba and co-workers translate into $0.055 \mathrm{TPa} \mathrm{nm}$ for the monolayer (roughly half our value). The origin of this large discrepancy is unclear, although it might be related to the interactions with neighboring layers, which are not present in our case.

For a hexagonal two-dimensional crystal only two elastic constants are required, ${ }^{42}$ and due to the underlying crystal symmetry, we obtain the following rule:

$$
C_{x x x x}=C_{x x y y}+2 C_{x y x y} .
$$

This provides an internal consistency check for our results, which as can be seen from the results in Table I, is satisfactorily obeyed [the numerical value obtained for $C_{x x x x}$ using $C_{x x y y}$ and $C_{x y x y}$ in Eq. (4) is within $3 \%$ of its calculated value, given in Table I]. A second consistency check is provided by the comparison of these values of the elastic constants with those obtained from the sound velocities reported above using the identities

$$
v_{L A}^{2 D}=\sqrt{\frac{C_{x x x x}}{\rho}}, \quad v_{T A}^{2 D}=\sqrt{\frac{C_{x y x y}}{\rho}},
$$

where $\rho$ is the surface mass density of the sheet. These data are also given in Table I. As can be seen there, the values obtained in these different fashions are in rather good agreement. From the elastic constants we can also calculate ${ }^{42}$ the Poisson ratio

$$
\sigma=\frac{C_{x x y y}}{C_{x x x x}}
$$

and Young's modulus

$$
Y=\frac{C_{x x x x}^{2}-C_{x x y y}^{2}}{C_{x x x x}} .
$$

The values obtained for $\sigma$ and $Y$ are also given in Table I. These values can be compared with those obtained previously for BN nanotubes using the same TB model; ${ }^{27}$ the value of $\sigma$ obtained here is a little bit larger than that obtained for the nanotubes $(0.263)$, but the agreement with the tube Young's modulus $(0.298-0.310 \mathrm{TPa} \mathrm{nm}$ depending on the tube diameter) is nearly perfect. We also point out that the inclusion of the dipolar interactions does not affect the values of the sound velocities or elastic constants, as expected from the fact that they only influence the highfrequency optical phonon bands, but not the acoustic ones, as can be seen in Fig. 2.

At the zone edge the ZA band corresponds mostly to $\mathrm{N}$-atom out-of-plane displacements, while the $\mathrm{ZO}$ mode corresponds to $\mathrm{B}$ displacements. This is most clearly seen in Figs. 2(b) and 2(c), which show the partial vibrational density of states (DOS) separated into its in-plane and out-ofplane atomic contributions.

The frequency of the Raman and infrared-active (IR) modes in bulk $h$-BN has been investigated by several authors $^{35,43-45}$ obtaining similar results. The values reported for the high-energy Raman-active $E_{2 g}$ mode are 1366 (Refs. 44 and 43), 1367 (Ref. 45), and 1370 (Ref. 35) $\mathrm{cm}^{-1}$. For the IR modes the values given in the literature are 1367 (Ref. 35 ) and $1383 \pm 5$ (Ref. 45) $\mathrm{cm}^{-1}$ for the $E_{1 u}$ (TO) mode, 1610 (Ref. 35) $\mathrm{cm}^{-1}$ for the $E_{1 u}(\mathrm{LO})$ mode, and $770 \pm 3$ (Ref. 45) and 783 (Ref. 35) $\mathrm{cm}^{-1}$ for the $A_{2 u}$ (TO) mode. At this point it is important to note that, since our calculations refer to a freestanding (isolated) flat sheet of $h$-BN rather than to the bulk material, the comparison with these experimental observations has to be made with some care. In analogy to the case of graphite and assuming relatively weak interlayer interactions, the frequencies of a single $\mathrm{BN}$ sheet should be closely related to those of the bulk. However, there is an important exception related to the role of the long-range electrostatic interactions. In a strictly $2 \mathrm{D}$ system like the one treated here (i.e., a sheet of monatomic thickness), the electrostatic interactions do not result in a macroscopic term at $\boldsymbol{\Gamma}$ for in-plane modes, and hence we do not obtain any LO-TO splitting in that limit (see the Appendix A). This striking difference is quite notorious, for example, comparing our phonon band structure in Fig. 2(a) with the calculations for bulk $h$-BN diplayed in Fig. 3 of Ref. 25. The absence of LO-TO splitting for the systems of reduced dimensionality considered here turns out to be quite important when considering the applicability of the zone-folding approach to the vibrational properties of $\mathrm{BN}$ nanotubes, a point to which we will return below. 
Our results for the planar $\mathrm{BN}$ sheet have, therefore, to be compared with the TO frequencies of the bulk. We obtain for the highest modes at $\boldsymbol{\Gamma}$ a frequency of $1446 \mathrm{~cm}^{-1}$, to be compared against the empirical values of the $E_{2 g}$ and $E_{1 u}(\mathrm{TO})$ modes, and $719 \mathrm{~cm}^{-1}$ for the $\mathrm{ZO}$ mode, to compare with the measurements for the $A_{2 u}(\mathrm{TO})$. The discrepancies are therefore smaller than $10 \%$, which we regard as acceptable, given the simplicity of the TB model employed in this work. It is noteworthy that the effect of the inclusion of the long-range electrostatic interactions improves the comparison with experiment, correcting the underestimation of the $\mathrm{ZO}(\boldsymbol{\Gamma})$ frequency and the overestimation of the $\mathrm{TO}(\boldsymbol{\Gamma})$ and $\operatorname{LO}(\boldsymbol{\Gamma})$ values, as well as giving a more realistic overbending of these modes along $\boldsymbol{\Gamma}-\mathbf{M}$ and $\mathbf{K}-\boldsymbol{\Gamma}$. We should emphasize here that no empirical information on vibrational or structural properties has been used in the construction and parametrization of the model. ${ }^{29}$ As pointed out above, it is also important to take into account that at least some of the discrepancy may not be attributed to the model, but to the geometry used in the supercell calculation. The presence of nearby sheets in the experiment is likely to soften somewhat the in-plane LO and TO modes, while hardening the $\mathrm{ZO}$ mode, which could account for some of the discrepancy between our results and the experimental ones. Discrepancies of similar size also occur between the first-principles values of Miyamoto et al. $^{23}$ and the experimental values. The overall topology of our phonon band structure is in good agreement with the results of Miyamoto et al., although we cannot make a direct comparison due to the unusual shape of the $h$-BN supercell used in that work. Kern et al. ${ }^{25}$ have also performed first-principles density-functional calculations of the phonon properties of both cubic and $h$-BN. Our phonon band structure is in reasonable agreement with that obtained by them, bearing in mind the different methodologies and geometries used in their work and ours.

Rokuta et $a l .{ }^{46}$ have obtained the phonon spectra of a BN monolayer deposited over a series of metal surfaces $(\mathrm{Ni}, \mathrm{Pd}$, and $\mathrm{Pt}$ ) using high-resolution electron-energy-loss spectroscopy. Again, there is overall good agreement between the results reported by Rokuta and co-workers and our own. Interestingly, they find no splitting at $\boldsymbol{\Gamma}$ between the LO and TO modes when the BN monolayer is placed over a $\mathrm{Ni}(111)$ surface, but some splitting is seen when the other metals are used. This result was considered as quite surprising by these authors, especially taking into account that $\mathrm{Ni}(111)$ was the sole substrate on which BN formed well-ordered commensurate monolayers, and it was suggested that this is because $\mathrm{Ni}$ can effectively screen the polarization field of the LO mode, while Pd and Pt cannot. However, we maintain that the polarization field only arises in 3D systems; in the 2D case the electrostatic interactions do not give rise to a macroscopic field for in-plane modes-i.e., the field has zero component at $\boldsymbol{\Gamma}$-and hence there is no splitting of the TO and LO bands in that limit. However, the electrostatic interactions do give rise to nonzero components of the field in regions of the Brillouin zone away from $\boldsymbol{\Gamma}$, and these result in a higher overbending when the electrostatic interactions are included. Another consequence of the inclusion of the electrostatic in- teractions is the fact that the LO mode approaches $\boldsymbol{\Gamma}$ with a finite slope, which does not occur when the electrostatics are not included [see Fig. 2(a)].

Prompted by this experimental study, we have considered the effect of the presence of an ideal metal surface in the proximity of the $\mathrm{BN}$ layer on the phonon properties of the latter. The metal surface is simply considered as a medium which generates dipolar images of the BN monolayer. The net result is to effectively shorten the range of the electrostatic interactions: the finite slope of the LO mode at $\Gamma$ is lost, and although quadrupolar interactions persist in the $\mathrm{BN}$ metal surface system, the dipolar interactions are screened out.

Interestingly, Rokuta et ll. $^{46}$ report a degeneracy of the $\mathrm{ZO}$ and ZA modes at $\mathbf{K}$. This is unexpected, because such modes should display a splitting related to the different masses of B and $\mathrm{N}$. The fact that no splitting is observed in the experiments is probably a result of a deviation from planarity in the $\mathrm{BN}$ monolayer due to the presence of the metal surface. Another important point might be the quite different positions occupied by $\mathrm{B}$ and $\mathrm{N}$ atoms on the surface. In fact, Rokuta and co-workers reported a buckling of the BN monolayers on $\mathrm{Ni}(111)$, where B atoms, which occupy fcc sites, lie $0.2 \AA$ below the $\mathrm{N}$ atoms, which are positioned directly on top of the $\mathrm{Ni}$ atoms in the surface.

\section{B. Nanotubes}

Let us now move on to discuss the results obtained for $\mathrm{BN}$ nanotubes. We have considered a series of $(n, n)$ (armchair), $(n, 0)$ (zigzag), and $(n, m)(n \neq m \neq 0$, chiral $)$ nanotubes. For $(n, 0)$ nanotubes we have included all tubes with $n$ from 6 to 16, i.e., diameters from 5 to $14 \AA$, approximately, while for $(n, n)$ tubes we considered specifically the $(6,6)$, $(10,10)$, and $(15,15)$ tubes, with diameters between 8 and 21 $\AA$. Only two chiral nanotubes were considered, since these structures usually have very large unit cells, namely, the $(10,5)$ and $(10,7)$ tubes, with diameters of 10.6 and $11.9 \AA$, respectively.

In Fig. 3, for the $(10,10)$ tube, and Fig. 4, for the $(14,0)$, we compare the phonon dispersion curves calculated directly from the tube structure and those obtained for the same tubes employing the zone-folding approach from the phonon bands of the flat sheet discussed above. First, let us remark that the zone-folding method cannot reproduce certain low-energy modes; in particular, it cannot give rise to a breathing mode, which in the flat sheet corresponds to a zero-energy translation perpendicular to the plane. However, the high-frequency modes are expected (and indeed are) to be reasonably well described. In spite of the limitation regarding low-frequency modes, the zone-folding approach has been extensively used for the interpretation of experimental results of carbon nanotube vibrational modes, ${ }^{14,36}$ partly due to its simplicity and also to the fact that the phonon modes of graphite can be determined with very high accuracy. One of the aims of the present paper is to study if the zone folding approximation can also be useful in the case of the BN nanotubes.

Generally speaking, for experimentally relevant tube diameters, the zone-folding method reproduces quite well the 
$(10,10)$

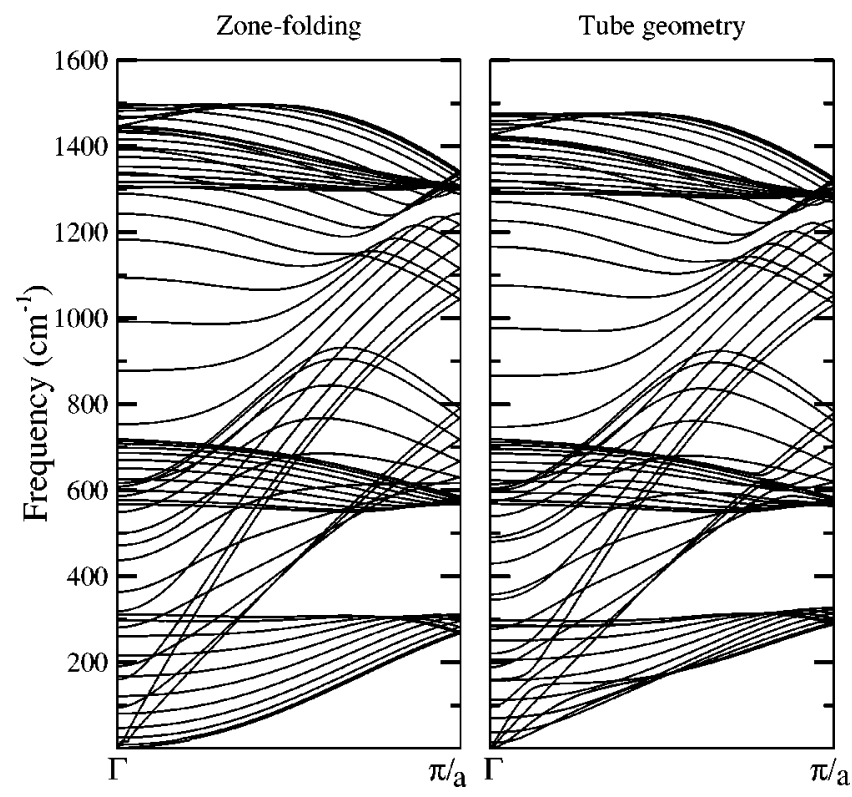

FIG. 3. Comparison of the the phonon dispersion curves of the $(10,10)$ tube (40 atoms in the unit cell and a diameter of $13.9 \AA$ ) calculated directly from the tube structure and those obtained using the zone-folding approach from the results for the flat sheet shown in Fig. 2(a).

overall phonon band structure. Deviations can be larger for the smaller nanotubes, where the effects of curvature are more noticeable. In particular, we shall see below how curvature effects influence the breathing mode. Another difference that can be appreciated between the tube dispersion bands and those determined from zone-folding is a general softening of frequencies for the explicit calculation, where curvature effects are taken into account. The downward shift is especially clear at high frequencies, but is also noticeable at intermediate ones. This shift is not homogeneous, and it has also a nontrivial dependence on the wave vector and on the nature of the mode (radial or tangential).

Also shown in Fig. 4 is the vibrational DOS for the $(14,0)$ tube decomposed in the different directions of the atomic displacements. This curve resembles closely the result obtained for the BN monolayer. There is a clear energy separation between radial and tangential modes (parallel or perpendicular to the tube axis), the radial modes corresponding to frequencies below $\sim 700 \mathrm{~cm}^{-1}$. This corresponds to the already mentioned separation between out-of-plane and inplane modes in the case of the planar sheet, as could be expected, since the radial modes are roughly derived from the out-of-plane vibrations of the plane.

There are four acoustic bands for the tubes. They correspond to a longitudinal mode (atomic displacements parallel to the tube axis), two degenerate transversal modes (atomic displacements perpendicular to the tube axis), and a torsion of the tube around its axis (the so-called twiston band which, in the limit of $\mathbf{k}=0$, generates a rigid rotation of the tube around its axis). This last mode is characteristic of a onedimensional system like the nanotubes, and no analog can be found for the bulk or the infinite planar sheet. According to
$(14,0)$
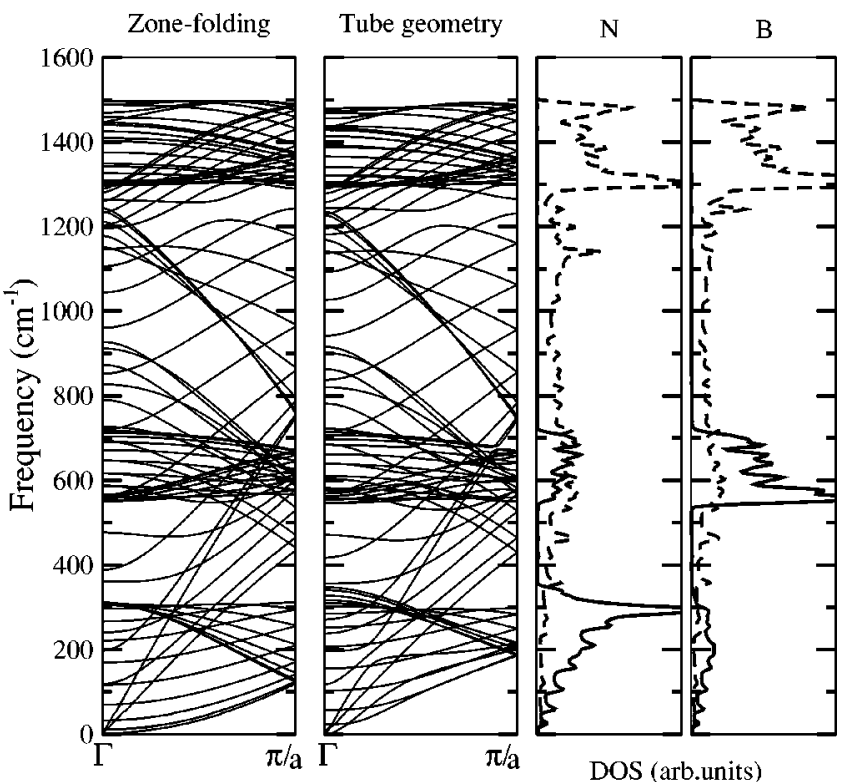

FIG. 4. Same as Fig. 3 for the $(14,0)$ tube (56 atoms in the unit cell and a diameter of $11.3 \AA$ ). The vibrational density of states decomposed in the different directions of the atomic displacements is also shown. Solid lines for out-of-plane displacements and dashed lines for in-plane displacements.

Saito et $a l .{ }^{47}$ and in contrast to the case of the flat sheet, all four bands would be required to approach $\boldsymbol{\Gamma}$ linearly. This relates to the fact that none of the acoustic bands of the nanotubes is solely derived from the quadratic out-of-plane acoustic band of the sheet: the transversal acoustic bands can be regarded as a combination of the TA in-plane and ZA out-of-plane modes of the planar sheet. However, it is interesting to point out here that the low-energy behavior of the transveral acoustic bands in the nanotubes is still the subject of some controversy and some authors have recently proposed that these bands should exhibit a quadratic behavior for small values of $\mathbf{k} .^{48,49}$ The situation is more clear for the other acoustic bands since it is possible to establish a oneto-one correspondence between the longitudinal and twiston bands of the tubes and the LA and TA bands of the planar sheet, respectively. In fact, the sound velocities of the latter bands are, within the precision of the calculations ( $\sim 1 \mathrm{~km} / \mathrm{s}$ ), independent of the tube radius and chirality, and almost identical (although somewhat smaller) to those of the corresponding planar-sheet bands. Similar results have been obtained for the case of the carbon nanotubes. ${ }^{47,50}$ This insensitivity to the tube structure and radius confirms the predictions of a simple continuum elasticity model of the tubes $^{51}$ where the elastic constants are directly taken from the planar sheet, from which one obtains $v_{L A}^{\text {tube }}=v_{L A}^{2 D}$ and $v_{\text {twiston }}^{\text {tube }}=v_{T A}^{2 D}$. The independence of the mechanical properties on the chirality of the tubes is here a simple consequence of the isotropy of the underlying hexagonal structure of the $\mathrm{BN}$ planes. We have used this model to estimate the sound velocity of the transversal band, obtaining $v_{T A}^{t u b e}=\sqrt{\frac{1}{2}} v_{T A}^{2 D}$ $=9 \mathrm{~km} / \mathrm{s}$. In the TB calculations, however, the transversal bands seem to be more sensitive to the numerical uncertain- 


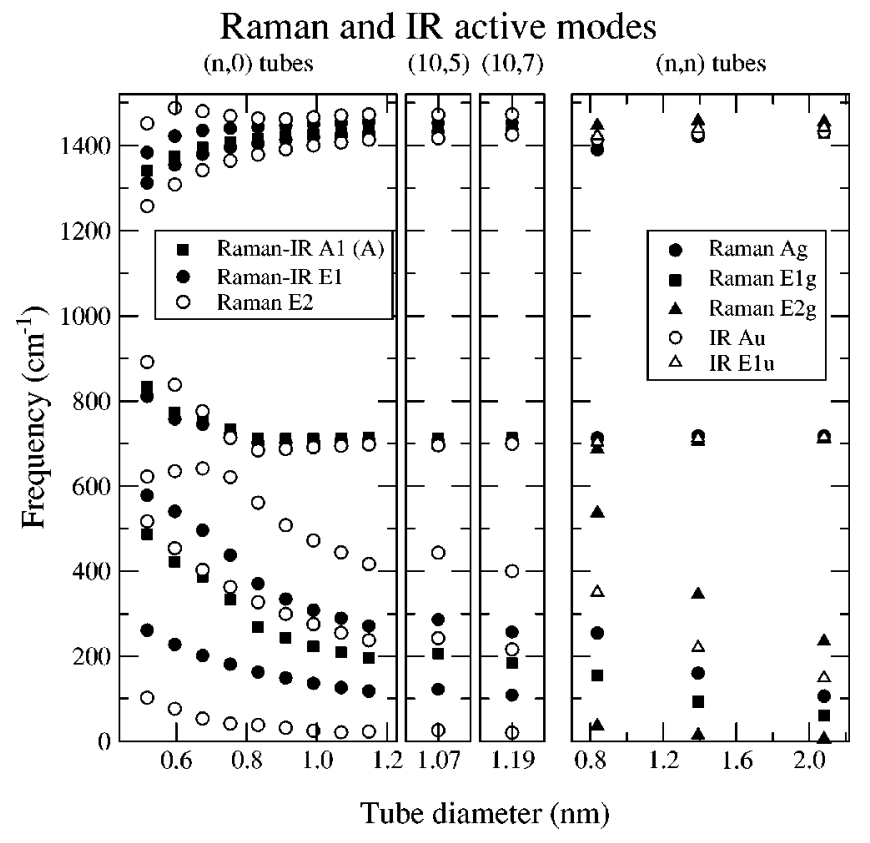

FIG. 5. Active modes for the $(n, 0)$, chiral and $(n, n)$ BN nanotubes considered in this work. Symbols have the same meaning for $(n, 0)$ and chiral tubes, except that for chiral tubes $A 1$ has to be read as $A$ (see the text). See Ref. 52 for the numerical values of the frequencies.

ties than the other acoustic bands, being difficult to extract an accurate slope. In fact, although the transversal bands apparently exhibit a linear dependence with the phonon wave vector (see Figs. 3 and 4), for very small values of $\mathbf{k}$, and consequently for very small frequecencies, they seem to bend slightly. This would be roughly consistent with the proposed $^{48,49}$ quadratic behavior, although problems of numerical accuracy cannot be ruled out. The clarification of this point will be the subject of future work. Here we have decided to make an estimation of the sound velocity of the transversal modes from the slope of these bands in the region where they still exhibit a clear linear behavior. In this way we have found values ranging from $7 \mathrm{~km} / \mathrm{s}$, for the narrower tubes, to $8 \mathrm{~km} / \mathrm{s}$, for those with larger diameters. This is again in reasonable agreement with the result that we obtained from continuum elasticity theory.

In Fig. 5 we have plotted the frequencies of the optically active modes for the different tubes considered as a function of the tube radius. ${ }^{52}$ The values plotted have been obtained including the long-range electrostatic interactions, but the picture does not change significantly if they are not included. ${ }^{53}$ We have classified the modes according to the corresponding symmetry point group, ${ }^{54}$ which for zigzag $(n, 0)$ nanotubes is $C_{2 n v}$, for armchair $(n, n)$ nanotubes is $C_{2 n h}$, and for chiral $(n, m)$ nanotubes is $C_{N}$, where $N$ $=2\left(n^{2}+m^{2}+n m\right) / d_{R}$ with $d_{R}$ being the greatest common divisor of $2 n+m$ and $2 m+n,{ }^{54}$ i.e., $N=70$ for the $(10,5)$ nanotube and $N=146$ for the $(10,7)$ nanotube. The total number of Raman- and IR-active modes and their distribution over the frequency spectrum are very similar for both zigzag and chiral nanotubes. In the $(n, 0)$ nanotubes the rotationally invariant modes can be classified according to how they transform under reflection through the symmetry plane $\sigma_{v}$, namely, $A_{1}$ and $A_{2}$ modes; only the first are Raman and IR active, the $A_{2}$ being inactive. The chiral tubes do not contain a $\sigma_{v}$ mirror plane, and therefore all six $A$ modes are active. In total, the $(n, 0)$ nanotubes have eight modes with nonzero frequency which are both IR and Raman active (three belong to the $A_{1}$ representation and five to the $E_{1}$ ) and six modes $\left(E_{2}\right)$ which are only Raman active, while for the chiral tubes the count is nine IR and Raman modes (four $A$ and five $\left.E_{1}\right)$ plus six $\left(E_{2}\right)$ Raman active modes. ${ }^{54}$ In the case of the $(n, n)$ nanotubes, due to the existence of inversion symmetry in the point group, the active modes can only be either IR or Raman active, having a total of four IR-active (one $A_{u}$ and three $E_{1 u}$ ) and nine Raman-active (three $A_{g}$, two $E_{1 g}$, and four $E_{2 g}$ ) modes. Comparing with carbon nanotubes, the bigger difference appears in the case of the $(n, 0)$ nanotubes, where the number of active modes is nearly double for BN nanotubes than for carbon nanotubes. ${ }^{55}$

In Fig. 6(a) we have plotted the frequency of the breathing mode as a function of the tube radius. This mode is of importance in carbon nanotubes because it can be correlated with the tube radius and it is Raman active for all tube chiralities. The same is true here. Although there are minor deviations at small radii (below $4 \AA$ ), the breathing mode frequency follows very closely a dependence on the inverse of the tube radius: $\nu_{B M}(r)=A / r$. This is corroborated in the $\log$ - $\log$ plot shown in the inset of Fig. 6(a), which fits very well to a linear behavior with a slope $-0.97 \pm 0.01(-1.09$ \pm 0.03 including the tubes with $r<4 \AA$ ). Leaving out the tubes with $r<4 \AA$, a fit to this expression gives $A$ $=1091 \mathrm{~cm}^{-1} \AA$, with a standard deviation smaller than 3 $\mathrm{cm}^{-1}$. This value is quite insensitive to the tube structure, as occurs also with carbon nanotubes. ${ }^{37,56}$ As can be seen in Fig. 6(a), the breathing mode exhibits a hardening for the smaller structures $(r<4 \AA)$ with respect to what would be expected from the $r^{-1}$ fit to the frequencies of the tubes with the larger diameters. If we include the frequencies of the smaller tubes, the quality of the fit decreases (mean deviation of $16 \mathrm{~cm}^{-1}$ ) and the value obtained for $A$ increases to 1160 $\mathrm{cm}^{-1} \AA$, in accordance with the mentioned hardening. Interestingly, this behavior is the oppositte to what was observed for the carbon tubes, ${ }^{37}$ where there is a softening of the breathing mode for increasing curvature. Therefore, this effect must be related to a unique characteristic of the $\mathrm{BN}$ tubes as compared to the carbon nanotubes: the buckled structure of its surface. For small- and moderate-radius BN nanotubes, the $\mathrm{B}$ atoms lie somewhat closer to the nanotube axis than the $\mathrm{N}$ atoms. This buckling is of the order of $0.1 \AA$ for the smallest nanotubes, but decreases rapidly with increasing radius. ${ }^{27}$ Figure 6 (b) shows how the nanotube atoms are displaced in the breathing mode. The values plotted are the magnitude of the radial displacement of $\mathrm{B}$ atoms $\left(\delta R_{B}\right)$ divided by that of the $\mathrm{N}$ atoms $\left(\delta R_{N}\right)$. For $\delta R_{B} / \delta R_{N}=1$ the displacements are of equal magnitude, and we would have a pure breathing mode, just as in a carbon nanotube. The observed behavior is slightly different: for tubes with $r<4 \AA$ the displacement of the $\mathrm{N}$ (outer) atoms is larger than that of the B (inner) atoms. This can be explained in a simple model 

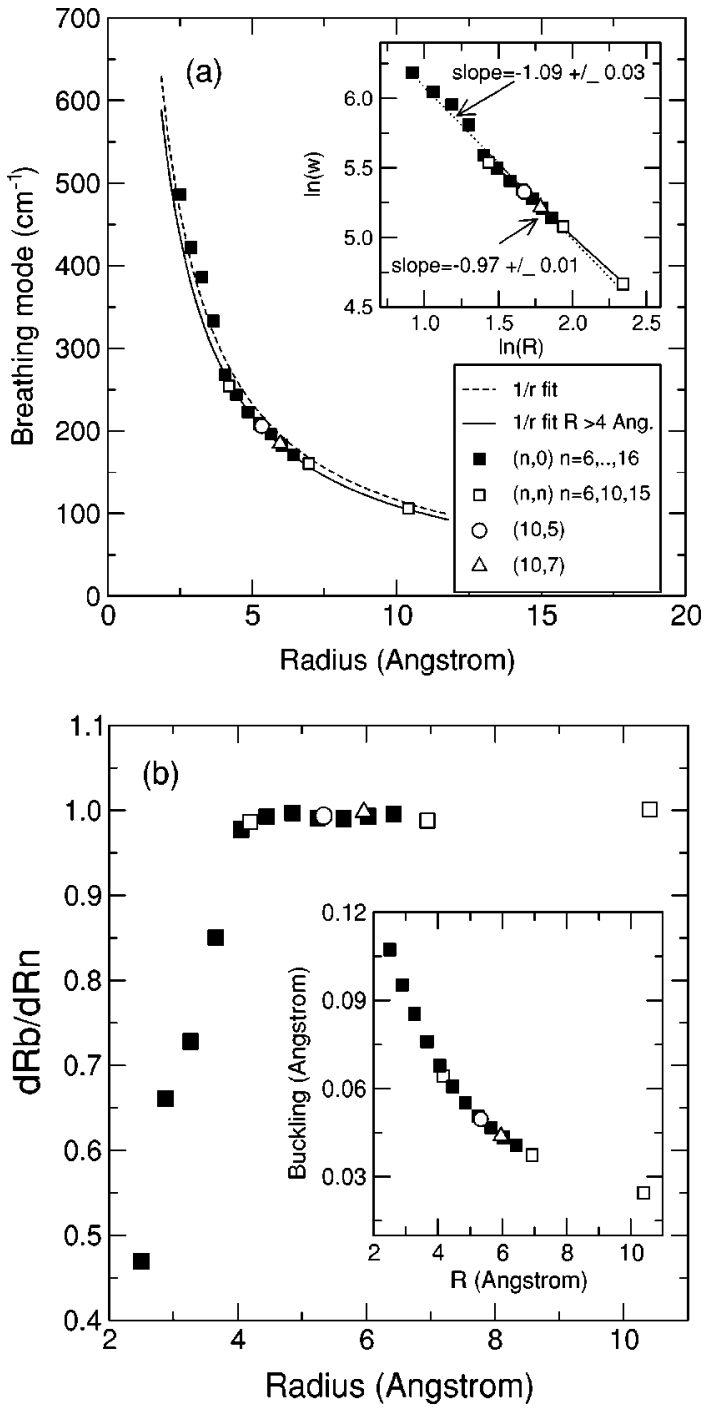

FIG. 6. (a) Frequency of the radial breathing mode of BN nanotubes as a function of tube radius. The inset presents a log-log plot which clearly shows the $r^{-1}$ behavior. Panel (b) shows the relative radial displacement of the $\mathrm{B}$ atoms and the $\mathrm{N}$ atoms in the radial breathing mode. The evolution of the buckling in the nanotube structure is plotted in the inset as a function of the radius. The meaning of the symbols is identical in all the panels and plots. See Ref. 52 for the numerical values of the frequencies.

as a consequence of the tendency to preserve in the pattern of displacements of this vibrational mode the bond angles of the buckled surface.

As is well known, ${ }^{24,48,56}$ the constant $A$ can be estimated from the elastic constants of the plane,

$$
A \approx \frac{1}{2 \pi} \sqrt{\frac{C_{x x x x}}{\rho}}=\frac{1}{2 \pi} v_{L A}^{2 D} .
$$

Using this equation we obtain the value $A=1135 \mathrm{~cm}^{-1} \AA$, which is in reasonable agreement with the value found by the direct calculation.

Finally, let us discuss the ability of the zone-folding approach to predict the frequencies of the active modes of $\mathrm{BN}$ nanotubes. In Figs. 7 and 8 we plot the frequencies obtained

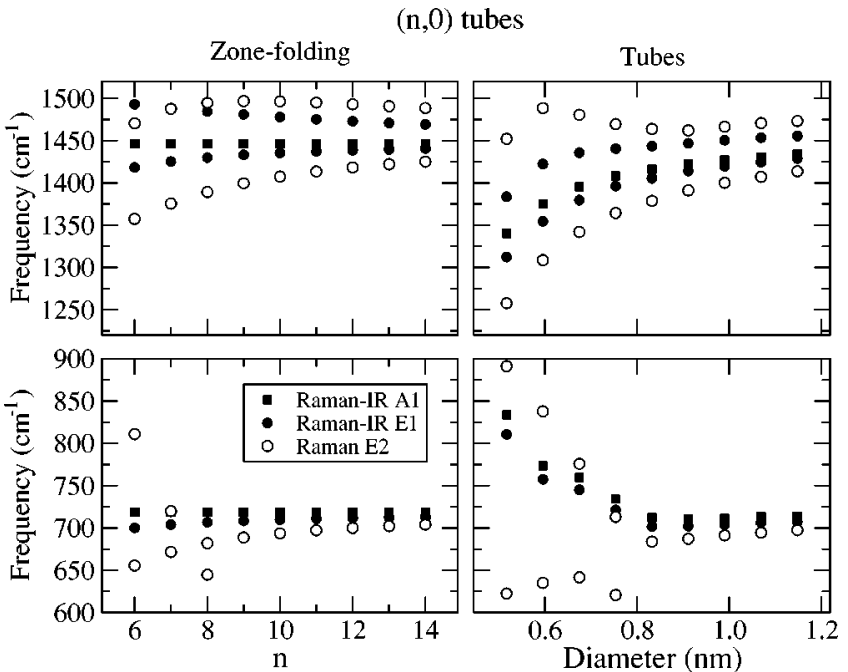

FIG. 7. Comparison between the optically active modes predicted by the zone-folding approximation and those obtained directly from the tubes geometry. Here we show the results for $(n, 0)$ BN tubes with $n$ running from 6 to 14. The modes have been classified according to their symmetry (the meaning of the symbols is the same in the four panels). For clarity, intermediate frequencies (around $700 \mathrm{~cm}^{-1}$ ) and high frequencies (above $1250 \mathrm{~cm}^{-1}$ ) are shown separately. See Ref. 52 for the numerical values of the frequencies.

from zone folding side by side with those obtained directly from the nanotubes, in both cases including the electrostatic interactions. For clarity, this is done separately for intermediate frequencies (around $700 \mathrm{~cm}^{-1}$ ) and for high frequencies (above $1250 \mathrm{~cm}^{-1}$ ) and separately for $(n, 0)$ and $(n, n)$ nanotubes. Overall, we can say that zone folding is capable of rather accurate estimations of the actual frequencies, particularly for nanotubes with radii larger than $4 \AA$. In this case even the ordering of the modes is accurately reproduced, both for $(n, 0)$ and $(n, n)$ tubes. The intermediate frequency modes are very well reproduced, but there is a slight overes-

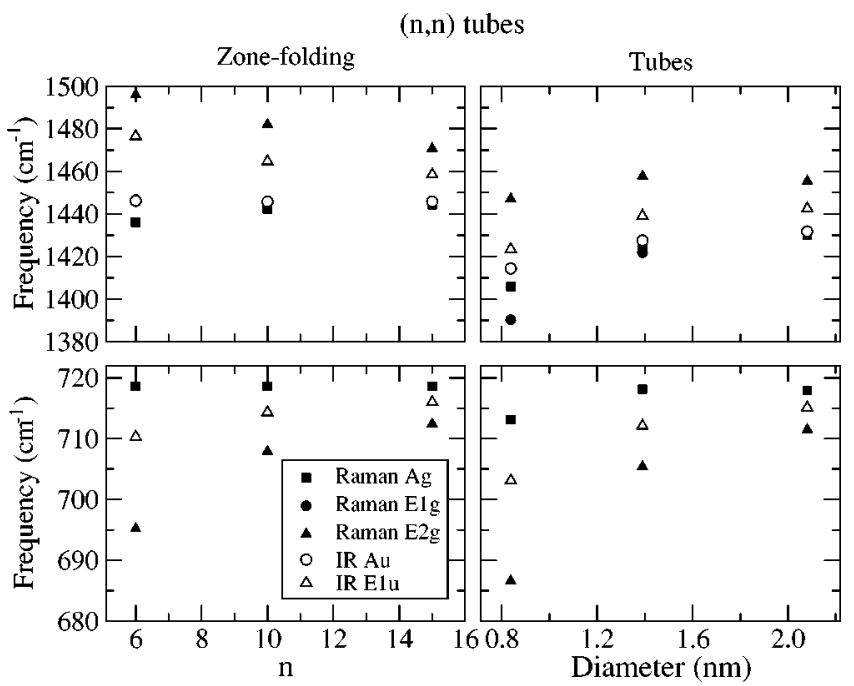

FIG. 8. Same as Fig. 7 but for $(n, n)$ tubes, with $n=6,10$, and 15. See Ref. 52 for the numerical values of the frequencies. 
timation of the high-frequency modes, of the order of a few tens of wave numbers.

That the zone-folding approach is capable of providing accurate estimates of the $\mathrm{BN}$ nanotube phonon frequencies rests in the absence of a $\mathbf{k}=0$ (macroscopic) dipolar field in the BN monolayer. The reduced dimensionality of the system prevents the buildup of a $\mathbf{k}=0$ component of the electrostatic field, which thus only has a finite range. Therefore, no splitting of modes is observed at $\boldsymbol{\Gamma}$; the only effect of the electrostatic interactions is to shift slightly the high-frequency modes and to confer a finite slope to the LO mode in the $\mathbf{k}$ $\rightarrow \boldsymbol{\Gamma}$ limit, modifications which do not prevent zone folding from providing accurate estimates of the phonon modes of isolated nanotubes. A subtle point here is that the applicability of zone folding in the case of $\mathrm{BN}$ also depends on the degree of transferability of the dielectric constant $\epsilon$ and the Born effective charges [see Eq. (1)] between the monolayer and nanotube geometries. For small nanotube radii there may be noticeable changes in the dielectric constant, but this is unlikely to affect the application of zone folding to all but the narrowest nanotubes.

\section{SUMMARY AND CONCLUSIONS}

In this work we have presented a detailed analysis of the vibrational properties of a $\mathrm{BN}$ monolayer and of singlewalled BN nanotubes of different diameters, including $(n, 0)$, $(n, n)$ and two chiral nanotubes. Our calculations are based on a nonorthogonal TB model complemented with longrange electrostatic interactions, not included in the TB model. The incorporation of these electrostatic interactions corrects to some extent the deficiencies of the TB model at intermediate and high frequencies in the $\mathrm{BN}$ layer. Using a simple model for a metal surface, we have analyzed the influence of placing a BN monolayer over such a surface on the phonon bands of the monolayer. The effect appears to be small and confined only to the high-frequency range, above $1250 \mathrm{~cm}^{-1}$. Generally, our calculated phonon frequencies, sound velocities, and elastic constants are in good agreement with previous experimental and theoretical results. Concerning the nanotubes, we have obtained phonon band structures and have analyzed the optically active modes (IR and Raman) as a function of the nanotube structure and radius. We have also performed a detailed comparison between the predictions of the zone-folding approach and the actual frequencies of optically active modes obtained directly from the nanotubes, finding rather good agreement between the two for all except the narrower tubes, having radii below $4 \AA$. High-frequency mode frequencies are systematically overestimated by about a few tens of wave numbers, but intermediate mode frequencies are given with high accuracy. Therefore, zone folding should also be useful in the context of BN nanotubes, as it has been in spectroscopic studies of carbon nanotubes. We also report on the behavior of the frequency and the pattern of atomic displacements of the radial breathing mode as a function of the tube radius.

\section{ACKNOWLEDGMENTS}

The authors want to thank Professor Pablo Ordejón for stimulating discussions. This work was supported in part by the Spanish Ministerio de Ciencia y Tecnología (Grant Nos. MAT2001-0946, BFM2000-1312-C02, and BFM200203278) and by the Basque Departamento de Educación, Universidades e Investigación, and the University of the Basque Country UPV/EHU (Grant No. 9/UPV 00206.215-13639/ 2001). D.S.P. acknowledges support from the Spanish Ministerio de Ciencia y Tecnología and CSIC under the "Ramón y Cajal" program. E.H. thanks the IST project SATURN (IST-1999-10593).

\section{APPENDIX ABSENCE OF LO-TO SPLITTING FOR A BN MONOLAYER}

In this appendix we briefly discuss the role of long-range electrostatic interactions in a monolayer, comparing the results with those found for bulk systems. Let us consider a thin slab of polar material parallel to the $x y$ plane. We focus first on the in-plane optical modes, for which the atomic displacements $\mathbf{u}$ are parallel to the $x y$ plane. Associated with this phonon there is an electric polarization field $\mathbf{P}=(1 / A)$ $\times(Z \mathbf{u}) f(z) \cos (\mathbf{k} \cdot \mathbf{r})$ and a charge density $\rho_{P}=-\nabla \cdot \mathbf{P}$, where $Z$ is the Born effective charge tensor within the unit cell, $A$ is the area of the unit cell, and $f(z)$ is a function related to the profile (number of layers) of the slab which can be approximated by $\delta(z)$ for a monolayer. We stress that $\mathbf{k}$, $\mathbf{u}$, and $\mathbf{P}$ are all parallel to the slab for pure in-plane modes. For the particular case of a system with hexagonal symmetry, like our BN sheets, the in-plane properties are isotropic, and $Z$ can be regarded as a scalar. Although this is not crucial for our arguments, we will make this simplification in the following. The charge density $\rho_{P}$ is proportional to the product $\mathbf{k} \cdot \mathbf{u}$, and no macroscopic charge accumulations appear due to the TO modes. However, a macroscopic electric field $\mathbf{E}_{\text {mac }}$ can appear associated with the LO modes. The correct limit when $\mathbf{k} \rightarrow 0$ is then calculated by solving Poisson's equation for $\rho_{P}$. The components of the electric field in the directions parallel to the slab are

$$
E_{\text {mac }}^{i}=\frac{4 \pi Z}{A \epsilon} \frac{(\mathbf{k} \cdot \mathbf{u})}{k} k^{i} B(z, k) \cos (\mathbf{k} \cdot \mathbf{r}),
$$

where $k$ is the modulus of $\mathbf{k}$ and $B(z, k)$ is given by

$$
B(z, k)=\int_{-\infty}^{\infty} d \alpha \frac{\bar{f}(\alpha k) e^{-i(\alpha k z)}}{1+\alpha^{2}}
$$

$\bar{f}(q)$ being the Fourier transform of $f(z)$. Once the electric field has been calculated, the corresponding modification of the force-constant matrix can be obtained taken into account that the force acting on an atom is given by $\mathbf{F}=Z \mathbf{E}_{\text {mac }}{ }^{33} \mathrm{In}$ the case of the monolayer we take $f(z)=\delta(z)$, then $B(z=0, k)=1 / 2$, and the correction of the force-contant matrix for the in-plane modes becomes

$$
C_{i j}=\frac{2 \pi Z^{2}}{A \epsilon} \frac{k^{i} k^{j}}{k} .
$$

This correction goes to zero when $\mathbf{k} \rightarrow 0$ irrespective of the polarization of the mode and, therefore, both LO and TO in-plane modes have the same frequency. This is in agreement with the results obtained for slabs of ionic materials 
using different models: ${ }^{57,58}$ pure in-plane optical modes have frequencies close to those of the TO phonons in the bulk. The linear behavior of the LO mode as it approaches $\Gamma$ can also be noticed. We move now to analyze the bulk limit. By taking $f(z)=1 / L, L$ being the distance between atomic layers, we can study the limit for bulk phonons with $k_{z}=0\left(k_{z}\right.$ is the wave vector perpendicular to the atomic layers in the bulk). $B(z, k)$ becomes now $1 /(L k)$, and the term in the force-constant matrix takes the usual form for bulk systems, ${ }^{33}$

$$
C_{i j}=\frac{4 \pi Z^{2}}{A L \epsilon} \frac{k^{i} k^{j}}{k^{2}}
$$

which has a different value for LO and TO modes. For the intermediate case of a slab with finite thickness we find bulk behavior for phonons with sufficiently large $k$, while the behavior of the monolayer is recovered when $k$ goes to zero. The slope of the LO modes at $\Gamma$ is proportional to the number of layers in the slab.

For modes with polarization vectors having components along the normal to the slab (out-of-plane components within the nomenclature used throughout this paper) things can be quite different. For these modes, $\mathbf{P}$ has a component along $z$ and, when $k=0$, the charge distribution $\rho_{P}$ associated with them can be rationalized as a number of infinite planes with charges of alternating signs along $z$. There are also surface charges given by $\mathbf{P} \cdot \hat{\mathbf{n}}=P_{z}$. All these charged planes give rise to electric fields which do not decay along the entire thickness of the slab. In fact, those modes with atomic displacements perpendicular to the slab, although are transversal by definition (since the only phonon wave vector that can be safely defined is parallel to the slab), are known to have frequencies similar to those of the corresponding longitudinal phonons in the bulk. ${ }^{57,58}$ For this reason, Fuchs and Kliewer $^{57}$ and Lucas ${ }^{58}$ arrived at the conclusion that the phonon-frequency distribution of an ionic crystal slab converges very rapidly to that of bulk. However, for anisotropic systems like $h$-BN, where the frequency range of the in-plane and out-of-plane vibrations is very different, the conclusion might be different.

In the case of a free monolayer the separation between in-plane and out-of-plane modes is exact for an arbitrary wave vector $\mathbf{k}$. There is only one out-of-plane optical mode (ZO) which, although its frequency is slightly modified by the long-range interactions, cannot exhibit any splitting since it is a nondegenerate mode. The LO and TO in-plane modes do not exhibit splitting as explained above.
*Electronic address: sqbsapod@sc.ehu.es

†Electronic address: ehe@icmab.es

${ }^{1}$ H. W. Kroto, J. R. Heath, S. C. O'Brien, R. F. Curl, and R. E. Smalley, Nature (London) 318, 162 (1985).

${ }^{2}$ S. Iijima, Nature (London) 56, 354 (1991).

${ }^{3}$ M. M. J. Treacy, T. W. Ebbesen, and M. J. Gibson, Nature (London) 381, 678 (1996).

${ }^{4}$ E. W. Wong, P. E. Sheehan, and C. M. Lieber, Science 277, 1971 (1997).

${ }^{5}$ A. Krishnan, E. Dujardin, T. W. Ebbesen, P. N. Yianilos, and M. M. J. Treacy, Phys. Rev. B 58, 14013 (1998).

${ }^{6}$ J.-P. Salvetat, G. A. Briggs, J.-M. Bonard, R. R. Bacsa, A. J. Kulik, T. Stöckli, N. A. Burnham, and L. AForró, Phys. Rev. Lett. 82, 944 (1999).

${ }^{7}$ W. A. de Heer, A. Châtelain, and D. Ugarte, Science 270, 1179 (1995); A. G. Rinzler, J. H. Hafner, P. Nikolaev, L. Lou, S. G. Kim, D. Tománek, P. Nordlander, D. T. Colbert, and R. E. Smalley, ibid. 269, 1550 (1995).

${ }^{8}$ H. Dai, J. H. Hafner, A. G. Rinzler, D. T. Colbert, and R. E. Smalley, Nature (London) 384, 147 (1996).

${ }^{9}$ C. Dekker, Phys. Today 52, 22 (1999).

${ }^{10}$ P. G. Collins, K. Bradley, M. Ishigami, and A. Zettl, Science 287, 1801 (2000); J. Kong, N. R. Franklin, C. Zhou, M. G. Chapline, S. Peng, K. Cho, and H. Dai, ibid. 287, 622 (2000).

${ }^{11}$ R. H. Baughman, A. A. Zakhidov, and W. A. de Heer, Science 297, 787 (2002); M. J. Biercuk, M. C. Llaguno, M. Radosavljevic, J. K. Hyun, A. T. Johnson, and J. E. Fishcher, Appl. Phys. Lett. 80, 2767 (2002); D. Quian, E. C. Dickey, R. Andrews, and T. Rantell, ibid. 76, 2868 (2000).

${ }^{12}$ P. M. Ajayan and T. W. Ebbesen, Rep. Prog. Phys. 60, 1025 (1997); P. M. Ajayan, Chem. Rev. 99, 1787 (1999); M. Terrones, W. K. Hsu, H. W. Kroto, and D. R. M. Walton, Top. Curr. Chem. 199, 189 (1999).
${ }^{13}$ M. S. Dresselhaus, G. Dresselhaus and P. C. Eklund, Science of Fullerenes and Carbon Nanotubes (Academic Press, New York, 1996); Carbon Nanotubes, Preparation and Properties, edited by T. W. Ebbesen (CRC Press, Boca Raton, 1997); P. J. Harris, Carbon Nanotubes and Related Structures (New Materials for the Twenty-First Century) (Cambridge University Press, Cambridge, England, 2001).

${ }^{14}$ M. S. Dresselhaus and P. C. Eklund, Adv. Phys. 49, 705 (2000).

${ }^{15}$ A. Jorio, A. G. Souza-Filho, G. Dresselhaus, M. S. Dresselhaus, A. K. Swan, M. S. Ünlü B. B. Goldberg, M. A. Pimenta, J. H. Hafner, C. M. Lieber, and R. Saito, Phys. Rev. B 65, 155412 (2002).

${ }^{16}$ A. Jorio, R. Saito, J. H. Hafner, C. M. Lieber, M. Hunter, T. McClure, G. Dresselhaus, and M. S. Dresselhaus, Phys. Rev. Lett. 86, 1118 (2001).

${ }^{17}$ A. Rubio, J. L. Corkill, and M. L. Cohen, Phys. Rev. B 49, 5081 (1994).

${ }^{18}$ X. Blase, A. Rubio, S. G. Louie, and M. L. Cohen, Europhys. Lett. 28, 335 (1994).

${ }^{19}$ N. G. Chopra, R. J. Luyken, K. Cherrey, V. H. Crespi, M. L. Cohen, S. G. Louie, and A. Zettl, Science 269, 966 (1995); A. Loiseau, F. Willaime, N. Demoncy, G. Hug, and H. Pascard, Phys. Rev. Lett. 76, 4737 (1996); M. Terrones, W. K. Hsu, H. Terrones, J. P. Zhang, S. Ramos, J. P. Hare, R. Castillo, K. Prasides, A. K. Cheetham, H. W. Kroto, and D. R. M. Walton, Chem. Phys. Lett. 259, 568 (1996); M. Terrones, A. M. Benito, C. Manteca-Diego, W. K. Hsu, O. I. Osman, J. P. Hare, D. G. Reid, H. Terrones, A. K. Cheetham, K. Prasides, H. W. Kroto, and D. R. M. Walton, ibid. 257, 576 (1996); K. Suenaga, C. Colliex, N. Demoncy, A. Loiseau, H. Pascard, and F. Willaime, Science 278, 653 (1997).

${ }^{20}$ L. Rapoport, Y. Bilik, Y. Feldman, M. Homyonfer, S. R. Cohen, and R. Tenne, Nature (London) 387, 791 (1997); Y. Feldman, E. 
Wasserman, D. J. Srolovitz, and R. Tenne, Science 267, 222 (1995); R. Tenne, L. Margulis, M. Genut, and G. Hodes, Nature (London) 360, 444 (1992).

${ }^{21}$ Y. Li, J. Wang, Z. Deng, Y. Wu, X. Sun, D. Yu, and P. Yang, J. Am. Chem. Soc. 123, 9904 (2001).

${ }^{22}$ R. S. Lee, J. Gavillet, M. Lamy de la Chapelle, A. Loiseau, J.-L. Cochon, D. Pigache, J. Thibault, and F. Willaime, Phys. Rev. B 64, 121405 (2001).

${ }^{23}$ Y. Miyamoto, M. L. Cohen, and S. G. Louie, Phys. Rev. B 52, 14971 (1995).

${ }^{24}$ K. N. Kudin, G. E. Scuseria, and B. I. Yakobson, Phys. Rev. B 64, 235406 (2001).

${ }^{25}$ G. Kern, G. Kresse, and J. Hafner, Phys. Rev. B 59, 8551 (1999).

${ }^{26}$ N. Ohba, K. Miwa, N. Nagasako, and A. Fukumoto, Phys. Rev. B 63, 115207 (2001).

${ }^{27}$ E. Hernández, C. Goze, P. Bernier, and A. Rubio, Phys. Rev. Lett. 80, 4502 (1998).

${ }^{28}$ For a review of tight binding see C. M. Goringe, D. R. Bowler, and E. Hernández, Rep. Prog. Phys. 60, 1447 (1997).

${ }^{29}$ J. Widany, T. Frauenheim, T. Köhler, M. Sternberg, D. Porezag, G. Jungnickel, and G. Seifert, Phys. Rev. B 53, 4443 (1996).

${ }^{30}$ D. Porezag, T. Frauenheim, T. Köhler, G. Seifert, and R. Kaschner, Phys. Rev. B 51, 12947 (1995).

${ }^{31}$ M. Elstner, D. Porezag, G. Jungnickel, J. Elsner, M. Haugk, T. Frauenheim, S. Suhai, and G. Seifert, Phys. Rev. B 58, 7260 (1998).

${ }^{32}$ P. Ordejón, D. A. Drabold, R. M. Martin, and S. Itoh, Phys. Rev. Lett. 75, 1324 (1995); W. Frank, C. Elsässer, and M. Fähnle, ibid. 74, 1791 (1995).

${ }^{33}$ X. Gonze, J.-C. Charlier, D. C. Allan, and M. P. Teter, Phys. Rev. B 50, R13 035 (1994); X. Gonze and C. Lee, ibid. 55, 10355 (1997).

${ }^{34}$ For the construction of the Born effective charge tensor in the case of the tube geometry we have identified purely radial and tangential displacements with, respectively, out-of-plane and inplane movements in the planar sheet. Therefore, for an atom $i$, which belongs to a tube of radius $r$ and axis $(0,0, z)$, and occupies the position $\left(r \cos (\theta), r \sin (\theta), z_{i}\right)$, the Born effective charge tensor takes the form

$$
Z_{\mu \nu}^{i}=\left(\begin{array}{ccc}
\left(Z_{\perp}^{i} \cos ^{2}(\theta)+Z_{\|}^{i} \sin ^{2}(\theta)\right) & \left(Z_{\perp}^{i}-Z_{\|}^{i}\right) \cos (\theta) \sin (\theta) & 0 \\
\left(Z_{\perp}^{i}-Z_{\|}^{i}\right) \cos (\theta) \sin (\theta) & \left(Z_{\perp}^{i} \sin ^{2}(\theta)+Z_{\|}^{i} \cos ^{2}(\theta)\right) & 0 \\
0 & 0 & Z_{\|}^{i}
\end{array}\right) .
$$

${ }^{35}$ R. Geick, C. H. Perry, and G. Ruppercht, Phys. Rev. 146, 543 (1966).

${ }^{36}$ A. Kasuya, Y. Sasaki, Y. Saito, K. Tohji, and Y. Nishina, Phys. Rev. Lett. 78, 4434 (1997).

${ }^{37}$ D. Sánchez-Portal, E. Artacho, J. M. Soler, A. Rubio, and P. Ordejón, Phys. Rev. B 59, 12678 (1999).

${ }^{38}$ In Ref. 24 some evidence was presented that, while for the small and moderate radii the strain energy is clearly smaller in the case of $\mathrm{BN}$ compared to $\mathrm{C}$ nanotubes, the opposite could be true for larger tubes ( $r>30 \AA$ ). However, this seems quite unlikely if, as pointed out here, a smaller flexular rigidity of $\mathrm{BN}$ can already be deduced from the behavior of the ZA phonon band of the flat sheet. Unfortunately, the parameter $\delta$ is quite sensitive to the fit procedure and to numerical uncertainities, and the values for $\mathrm{C}$ and $\mathrm{BN}$ are close enough so that no definitive conclusion can be given here.

${ }^{39}$ N. W. Ashcroft and N. D. Mermin, Solid State Physics (Saunders, Philadelphia, 1976).

${ }^{40}$ C. Kittel, Introduction to Solid State Physics (Wiley, New York, 1986).

${ }^{41}$ In fact, the in-plane stiffness $C$ reported in Ref. 24 should be compared with our Young modulus $Y$ (Table I). The elastic constant $C_{x x x x}$ is, in the notation of that reference, given by $C /(1$ $-\nu^{2}$ ) with a value of $0.284 \mathrm{TPa} \mathrm{nm}$ and $\nu$ being the Poisson ratio.

${ }^{42}$ L. D. Landau and E. M. Lifshitz, Theory of Elasticity, Course of Theoretical Physics, Vol. 7 (Pergamon Press, Oxford, 1986).

${ }^{43}$ T. Kuzuba, K. Era, T. Ishii, and T. Sato, Solid State Commun. 6, 523 (1968).

${ }^{44}$ R. J. Nemanich, S. A. Solin, and R. M. Martin, Phys. Rev. B 23, 6348 (1981).

${ }^{45}$ D. M. Hoffman, G. L. Doll, and P. C. Eklund, Phys. Rev. B 30, 6051 (1984).

${ }^{46}$ E. Rokuta, Y. Hasegawa, K. Suzuki, Y. Gamou, C. Oshima, and A. Nagashima, Phys. Rev. Lett. 79, 4609 (1997).

${ }^{47}$ R. Saito, T. Takeya, T. Kimura, G. Dresselhaus, and M. S. Dresselhaus, Phys. Rev. B 57, 4145 (1998).

${ }^{48}$ G. D. Mahan, Phys. Rev. B 65, 235402 (2002).

${ }^{49}$ V. N. Popov, V. E. Van Doren, and M. Balkanski, Phys. Rev. B 61, 3078 (2000).

${ }^{50}$ J. Yu, R. K. Kalia, and P. Vashista, J. Chem. Phys. 103, 6697 (1995).

${ }^{51}$ B. I. Yakobson, C. J. Brabec, and J. Bernholc, Phys. Rev. Lett. 76, 2511 (1996).

${ }^{52}$ See EPAPS Document No. E-PRBMDO-66-235415 for the tables containing the numerical values of the vibrational frequencies at $\boldsymbol{\Gamma}$, including the Raman- and IR-active modes, for all the tubes studied in this work. The frequencies have been classified according to their symmetries. A direct link to this document may be found in the online article's HTML reference section. The document may also be reached via the EPAPS homepage (http:// www.aip.org/pubservs/epaps.html) or from ftp.aip.org in the directory /epaps/. See the EPAPS homepage for more information.

${ }^{53}$ Similarly to the case of the monolayer, the main changes on the phonon band structure of the single-walled tubes induced by the long-range dipolar interactions are relatively small and restricted to the high-frequency modes: the in-plane optical modes extend over a somewhat larger energy range, and some of the longitudinal modes (with atomic displacements parallel to the tube axis) exhibit a larger dipersion with $\mathbf{k}$. These changes are directly related to the larger overbending of the LO band of the $\mathrm{BN}$ monolayer when the electrostatic interactions are included.

${ }^{54}$ O. E. Alon, Phys. Rev. B 64, 153408 (2001).

${ }^{55}$ O. E. Alon, Phys. Rev. B 63, 201403 (2001).

${ }^{56}$ J. Kürti, G. Kresse, and H. Kuzmany, Phys. Rev. B 58, R8869 (1998).

${ }^{57}$ R. Fuchs and K. L. Kliewer, Phys. Rev. 140, A2076 (1965).

${ }^{58}$ A. A. Lucas, J. Chem. Phys. 48, 3156 (1968). 